\title{
On the Possibility of Binding of Two Electrons to Dipole Potentials
}

\author{
PIOTR SKURSKI, ${ }^{1,2}$ MACIEJ GUTOWSKI, ${ }^{3}$ JACK SIMONS ${ }^{1}$ \\ ${ }^{1}$ Department of Chemistry, University of Utah, Salt Lake City, Utah 84112 \\ ${ }^{2}$ Department of Chemistry, University of Gdańsk, 80-952 Gdańsk, Poland \\ ${ }^{3}$ Materials and Chemical Sciences, Pacific Northwest National Laboratory, Richland, \\ Washington 99352
}

Received 7 October 1998; revised 17 November 1998; accepted 23 November 1998

\begin{abstract}
The possibility of binding two electrons by the fixed finite dipole (FFD) potential due to two point charges $+q e$ and $-q e$ separated by the distance $R$ is explored at the full configuration interaction level with extended basis sets. The critical value of the dipole moment $\mu=q R$ required to bind two electrons tends to infinity for small $q$ $(q \approx 0.91 e)$ and decreases precipitously as $q$ increases. In the limit of very large $q$ (and small $R$ ), this critical dipole moment seems to approach a limit below 2 Debyes (D). It is shown analytically that in the point dipole limit this critical dipole value will approach that for binding a single electron. An extension of the FFD model to include effects of inner-shell core electrons allows the $\mathrm{Li}^{-}, \mathrm{Na}^{-}$, and $\mathrm{K}^{-}$cases (with a $-1 e$ charge at $R$ ) also to be examined. FFD-plus-core systems display even larger critical dipoles (113, 129 , and $141 \mathrm{D}$, respectively) than does the +qe/-qe FFD potential (92.2 D). These findings suggest that it will be difficult to find a real molecule that can bind (by $\approx 1 \mathrm{~cm}^{-1}$ ) two electrons via its dipole potential. Finally, a simple electrostatic model is introduced which permits the critical dipole value of the FDD and its core-orbital extension to be evaluated rather well. @ 2000 John Wiley \& Sons, Inc. Int J Quant Chem 76: 197-204, 2000
\end{abstract}

Correspondence to: J. Simons.

Contract grant sponsor: NSF.

Contract grant number: CHE9618904.

Contract grant sponsor: Polish State Committee for Scientific Research (KBN).

Contract grant number: 3 T09A 04915.

Contract grant sponsor: Office of Basic Energy Sciences, U.S. Department of Energy. 


\section{Introduction}

$\mathrm{t}$ was recognized long ago that dipole-bound singly charged anions can be electronically stable if the dipole moment $\mu$ of the neutral molecular host exceeds 1.625 Debyes (D).* Specifically, with dipole moments greater than this critical value, for a point dipole potential $(V=$ $\left.-\mu e \cos \theta / r^{2}\right)$, there are an infinity of bound states within the context of the Born-Oppenheimer (BO) approximation (i.e., when the dipole is not rotating). It has also been shown that the same critical dipole moment that applies to the point dipole potential also applies to fixed (i.e., nonrotating) finite dipoles (FFD), even in the presence of a short-range repulsive core potential [2-4]. In fact, although the electron binding energies of the point or FFD models for $\mu>\mu_{\text {crit }}$ depend on the repulsion potential and the dipole moment magnitude, the value of the critical dipole does not, in the one-electron binding case.

It is important to distinguish these earlier studies from the point of view taken in the present work. Our goal was to determine under what conditions a realistically measurable level of electron binding can occur for two electrons. We define this binding threshold to be $\approx 1 \mathrm{~cm}^{-1}$ because such weak binding is within the realm of current (or near-term) experimental capability. The earlier analytical work and its accompanying numerical finite-basis efforts [1] tried to determine the absolute limit for binding of one electron. This limit is $\mu_{\text {crit }}=1.625 \mathrm{D}$ at which infinitesimal binding occurs. At a value of $\mu=1.695 \mathrm{D}$ (see Turner et al. [1d]), the one-electron binding energy is $1.22 \times 10^{-18} \mathrm{eV}$. Clearly, with our pragmatic definition of critical binding $\left(\approx 1 \mathrm{~cm}^{-1}\right)$, we will not be able to approach the absolute critical binding conditions, but this was not our goal.

Because numerical calculations using the FFD model (i.e., stationary $+q$ and $-q$ charges separated by a distance $R$ such that $q R=\mu$ ) have proven very useful in supplementing the analytical work on the conditions for critical binding of a single electron, we chose to employ this approach

*Analytical rather than numerical approaches were put forth by Fermi and Teller [1a] as well as by Wightman [1b]. Early finite basis-set estimates to the critical dipole moment of ca. $2.1 \mathrm{D}$ were obtained by Wallis et al. [1c] and later refinement using better basis sets gave $1.695 \mathrm{D}$ [1d]. in the present effort on the possibility of binding two electrons (where analytical solutions are not feasible). However, our ultimate interest lies in determining when a "real" molecule can bind two electrons through its dipole potential. Because the point and FFD models are known to yield highly inaccurate binding energies for $\mu>\mu_{\text {crit }}$ (the former giving infinite binding, and the latter can grossly overestimate binding), we also introduce here in the subsection, The FFD-Plus-Core Model, a modification of the FFD potential that produces much more realistic estimates to binding energies when $\mu>\mu_{\text {crit }}$. This model, we feel, gives a better way to determine the two-electron critical binding conditions for real molecules.

As Jordan and Luken demonstrated, the loosely bound electron in a dipole-bound state occupies a diffuse orbital localized mainly on the positive side of the dipole [5]. The average distance between this electron and the neutral molecular core can be quite large (typically 10-100 ̊). Because the electron is far from the core and because the dominant potential binding the electron to the core is the charge-dipole electrostatic potential, it is often adequate to use the Koopmans' theorem (KT) approach [6] to calculate the electron binding energy for real molecular systems. This static approximation neglects both electron correlation and orbital relaxation effects. The latter have been found to be quite small for most dipole-bound anionic states [7-13]. On the other hand, it has been found that electron correlation often leads to a sizable stabilization of dipole-bound anions and, in some cases, even provides the dominant contribution to the electron binding energy [9-13]. Therefore, to achieve very accurate electron binding energies, it is usually best to employ correlated methods as we do in the present effort (where correlation involving the two extra electrons are expected to be very strong).

To the best of our knowledge, the possibility of binding two electrons to a FFD has not been resolved. Moreover, the attention of experimentalists and computational chemists who deal with multiply charged anions has been limited to valencebound species [14-18]. Many valence dianions that are well known in condensed phases are found to be electronically unbound in the gas phase because of the strong Coulomb repulsion between the excess electrons [18]. Most gas-phase-stable valence multiply charged anions overcome this repulsion by delocalizing the excess negative charges among several electronegative atoms. For example, in 
$\mathrm{TeF}_{8}^{2-}$ and $\mathrm{LaF}_{6}^{3-}$, the excess charges are delocalized over numerous ligands [19, 20].

Because of the growing interest in multiply charged anions [14-18], as well as our long-standing interest in dipole-bound singly charged anions, we decided to perform calculations on the FFD model systems (and the variant discussed in the subsection The FFD-Plus-Core Model) to determine the features of this electrostatic potential that are required to bind two electrons. Of course, the ultimate goal is to use the results from these model calculations to suggest realistic molecular systems that may be able to bind two electrons in this manner.

\section{Methods}

\section{HOW THE SECOND-ELECTRON BINDING ENERGY IS CALCULATED}

The electron binding energies were computed by subtracting the energy of the doubly charged anion from the energy of the singly charged anion. For the FFD potential, rather than reporting electron binding energies for various $q$ and $R$ values, we report the critical positions $R_{\text {crit }}$ for which the second electron is barely (i.e., $\approx 1 \mathrm{~cm}^{-1}$ ) bound for various $q$ values, from which we then compute the critical dipole as $\mu_{c r i t}=q R_{\text {crit }}$. We do this because we know from experience in applying the FFD model to singly charged anions that this model overestimates the magnitude of the binding energies (when compared to real many-electron molecules) but is quite capable of determining the critical binding conditions [5]. In fact, we remind the reader that the FFD model has exactly the same $1.625 \mathrm{D}$ critical dipole value, independent of $q$, as the point dipole model in the one-electron case, even if the potential also includes a repulsive short-range cutoff. When using the FFD-plus-core potential model detailed below, we used conventional atomic orbital basis sets (see Testing the Basis subsection) and an SCF-level treatment of the $\left(1 s^{2}, 1 s^{2} 2 s^{2} 2 p^{6}\right.$ and $\left.1 s^{2} 2 s^{2} 2 p^{6} 3 s^{2} 3 p^{6}\right)$ core orbitals. The one or two "extra" electrons and their orbitals were treated exactly as in the FFD potential case (see below).

The energies of the monoanion and dianion were determined using the configuration interaction (CI) method including all single and double excitations to treat one or two extra electrons (i.e., a full CI calculation), using the restricted
Hartree-Fock (RHF) reference function. For the one-electron monoanion, of course, only a single configuration is necessary, so the RHF calculation provides the final energy. For the dianion, we examined both the singlet and triplet states, and in every case, the singlet state proved to be the ground electronic state for geometries where the dianion is electronically bound.

\section{ATOMIC ORBITAL BASIS SETS}

The diffuse character of the outermost electrons' density necessitates the use of very flexible atomic orbital basis sets containing functions with very low exponents. In this work, we used an uncontracted aug-cc-pVQZ basis set [21] supplemented with diffuse $s, p$, and $d$ symmetry functions centered on the positive charge of the dipole. We used even-tempered six-term $s$, six-term $p$, and fourterm $d$ sets having a geometric progression ratio equal to 3.2 [22], and for every symmetry, we began the exponents of the extra diffuse functions from the lowest exponent of the same symmetry included in the aug-cc-pVQZ basis set designed for hydrogen. We thereby achieved the lowest exponents of $2.200715(-5), 7.897615(-5)$, and $1.811981(-3)$ for the $s, p$, and $d$ symmetries, respectively. In some of our calculations, the charge $q$ is larger than $1 e$, in which case, we scaled the Gaussian exponents by $q^{2}$ [23], after which the scaled basis set was supplemented with extra diffuse $s$ and $p$ functions with exponents forming an even-tempered progression starting from the lowest exponent in the scaled set and proceeding with a geometric constant of 5.0 until a most diffuse exponent of $10^{-5}$ is reached. This means, for example, that for the largest $q$ considered, $q=10,000 e$, we supplemented the scaled-and-supplemented aug-cc-pVQZ set with $12 s$ and $12 p$ additional diffuse functions, giving an atomic orbital basis containing 140 total functions.

\section{TESTING THE BASIS}

To test our basis, we explored the dependence of the electron binding energy on the choice of the extra diffuse functions for cases with $\mu$ slightly above $\mu_{\text {crit }}$. These tests were performed with the uncontracted aug-cc-pVQZ core basis set (corresponding to $q=1 e$ ) and with only the extra diffuse functions being varied. We found that for $q=1 e$ and $R=19.19 \AA$ when the second electron 
is barely ${ }^{\dagger}$ bound, the electron binding energy increased by less than $1 \mathrm{~cm}^{-1}$ after inclusion of two additional sets of diffuse $s$ and $p$ functions. Next, we replaced the $6 s p 4 d$ set of diffuse functions by a seven-term $s p$ and five-term $d$ diffuse set with the geometric progression ratio reduced to 2.4 and found the electron binding energy to again increase by less than $1 \mathrm{~cm}^{-1}$.

Next, we verified that our scaled-and-extended basis set reproduced reasonably well the energies needed to remove one electron from the two-electron atomic systems $\mathrm{H}^{-}, \mathrm{He}, \mathrm{Li}^{+}, \mathrm{Be}^{2+}$, and $\mathrm{B}^{3+}$. These tests were carried out because they arise in the FFD cases for $R \rightarrow \infty$ and $q=1-5$. Specifically, our calculated and the experimental results $(\mathrm{eV})$ are 0.749 versus 0.754 for $\mathrm{H}^{-}[24], \$ 24.571$ versus 24.580 for $\mathrm{He}$ [25], 75.616 versus 75.619 for $\mathrm{Li}^{+}$ [25], 153.848 versus 153.850 for $\mathrm{Be}^{2+}$ [26], and 259.297 versus 259.298 for $\mathrm{B}^{3+}$ [25].

We also considered how our scaled-and-extended basis set performs for one excess electron in the field of the FFD. Using our $\approx 1 \mathrm{~cm}^{-1}$ definition of binding, the critical value of $\mu$ required to bind one excess electron was computed to be 2.01, $1.95,1.84,1.80$, and $1.76 \mathrm{D}$ for $q$ equal to $1,10,100$, 1,000 , and $10,000 e$, respectively, which can be compared with the analytical result of $1.625 \mathrm{D}$ [1] and to the early numerical predictions of ca. 2.0 D [1]. Because $\mu_{\text {crit }}$ should be $1.625 \mathrm{D}$ for all $q$ values in this one-electron FFD case to achieve infinitesimal binding, these results suggest that our finite basis set performs better the closer the finite dipole is to a point dipole, although the fact that we achieve different critical dipoles for different $q$ values is at least partly a result of how we define critical binding. ${ }^{\dagger}$

In the cases discussed in the subsection The FFD-Plus-Core Model, we replaced the $+1 e$ point charge by a $+3 e,+11 e$, or $+19 e$ charge that we surround by 2,10 , or 18 electrons occupying the lowest available atomic orbitals. These calculations were performed to assess the effects of core orbitals on the critical binding ${ }^{\dagger}$ conditions for two

\footnotetext{
${ }^{\dagger}$ Realizing that we are carrying out numerical calculations using a finite basis set and keeping in mind that we are trying to determine under what conditions a molecule can bind two electrons by an amount that is small yet experimentally observable, we chose to define the binding limit to be $1 \mathrm{~cm}^{-1}$ or more when we consider it bound.

${ }^{\ddagger}$ Experimental result is $6082.99 \pm 0.15 \mathrm{~cm}^{-1}(0.754199 \mathrm{eV})$ by Lineberger et al. [24b].
}

electrons. In these calculations, the 2,10 , or 18 core electrons were described at the SCF level with a 6-311G basis set [26]. The diffuse part of the basis set used to describe the extra electrons was the same as discussed earlier. All calculations were carried out on a Silicon Graphic Power Challenge numerical server and an IBM RS/6000 3BT workstation using the Gaussian 94 [27] package.

\section{Results and Discussion}

\section{BINDING OF TWO ELECTRONS BY A POSITIVE +q CHARGE}

As the first step, we explored how small the charge of the positive nucleus can be and still bind two electrons, and we determined that the critical value is $q=+0.91161 e$. This result indicates that for a finite dipole built of two points charges $+q$ and $-q$, we cannot expect binding of two electrons if $q$ is smaller than $+0.91161 e$, even if the distance $R$ between the charges forming the dipole is infinitely large. We must therefore use the values $q>0.91161 e$ if we intend to build a system which is able to bind two electrons by electrostatic attraction to $\mathrm{a}+q$ and $\mathrm{a}-q$ charge separated by some finite distance $R$. This result shows that the twoelectron critical binding conditions for the FFD model are not identical to those for one-electron binding to the FFD because that latter has $\mu_{c r i t}=$ $1.625 \mathrm{D}$ for any $q$ value.

\section{THE FFD CASE}

We next considered various integer and fractional point charges $+q$ and $-q$ in the range $0.91161 e<q \leq 10,000 e$. We performed calculations for such large $q$ values because we wanted to extrapolate to the point-dipole limit by taking $q \rightarrow \infty, R \rightarrow 0$, with $q R$ finite. For each value of $q$, we determined the critical distance $R_{\text {crit }}$ between the point charges required to bind two electrons; for $R$ greater than this critical distance, two electrons will be bound by $>1 \mathrm{~cm}^{-1}$, but for $R$ smaller, one electron will detach (or be bound by $<1 \mathrm{~cm}^{-1}$ ). The values of $R_{\text {crit }}$ and the corresponding values of the dipole moments are given in Table I for the values of $q$ examined here. Clearly, the critical dipoles for the FFD model seem to depend very strongly on $q$, unlike the one-electron binding case, and to be extremely large for small $q$ (the most chemically relevant). 
TABLE I

Charges $q$ (in e), critical distances $R_{\text {crit }}$ (in $\AA$ ), and corresponding dipole moments $\mu$ (in Debye), required to bind two electrons; the approximate values of $\boldsymbol{R}_{\text {crit }}$ determined from Eq. (1) are given in parentheses.

\begin{tabular}{rcr}
\hline$q$ & $R_{\text {crit }}$ & $\mu$ \\
\hline 10000.00 & 0.0000410 & 1.9693 \\
1000.00 & 0.0004640 & 2.2287 \\
100.00 & 0.0050284 & 2.4153 \\
10.00 & 0.0754196 & 3.6226 \\
8.00 & 0.1016904 & 3.9075 \\
7.00 & 0.1222986 & 4.1120 \\
6.00 & 0.152282 & 4.3887 \\
5.50 & 0.172926 & 4.5683 \\
5.00 & 0.199387 & 4.7885 \\
4.50 & 0.234373 & 5.0659 \\
4.00 & 0.282498 & 5.4276 \\
3.50 & 0.352252 & 5.9218 \\
3.00 & 0.460990 & 6.6427 \\
2.50 & 0.649800 & 7.8029 \\
2.00 & 1.041550 & 10.0056 \\
& $(1.17)$ & \\
1.90 & 1.173610 & 10.7106 \\
1.80 & 1.338620 & 11.5735 \\
1.70 & 1.549780 & 12.6547 \\
1.60 & 1.828200 & 14.0501 \\
1.50 & 2.209900 & 15.9220 \\
& $(2.34)$ & \\
1.40 & 2.761120 & 18.5672 \\
1.30 & 3.618380 & 22.5939 \\
1.20 & 5.113200 & 29.4719 \\
& $(5.21)$ & \\
1.10 & 8.301800 & 43.8631 \\
1.00 & 19.190000 & 92.1742 \\
& $(19.23)$ & \\
0.95 & 47.000000 & 214.4646 \\
0.91161 & $\infty$ & $\infty$ \\
\hline & &
\end{tabular}

\section{AN APPROXIMATION TO $R_{\text {crit }}$ (AND THUS TO $\mu_{\text {crit }}$ )}

While examining the results of our ab initio calculations, we discovered that the value $R_{\text {crit }}$ obtained in the FFD calculation can be estimated by using a simple first-order electrostatic model in which the two-electron atom having nuclear charge $+q$ is destabilized by a distant point charge $-q$. If the destabilizing Coulomb interaction of one of the electrons of this atom or ion with the charge $-q$, given approximately by $q / R$, exceeds the electron binding energy $E A(q)$ of this atom or ion, then the second electron becomes electronically unbound.
This hypothesis leads to approximate expressions for $R_{\text {crit }}$ and $\mu_{\text {crit }}$ :

$$
R_{\text {crit }} \approx q / E A(q) ; \mu_{\text {crit }} \approx q^{2} / E A(q) .
$$

As shown in Table I, Eq. (1) appears to work reasonably well even for relatively large charges $q$, even though polarization of the two-electron atom or ion by the charge $-q$ as well as penetration effects in the first-order Coulomb interaction are neglected in Eq. 1.

\section{EXTRAPOLATION OF THE FFD TO $q \rightarrow \infty, \boldsymbol{R} \rightarrow \boldsymbol{0}$, WITH FINITE $q R$}

There clearly is a very strong dependence of the two-electron $\mu_{\text {crit }}$ on $q$ as shown in Table I. For $q=2$ and $3 e, \mu_{\text {crit }}$ drops to 10.01 and $6.64 \mathrm{D}$, respectively, and the corresponding $R_{\text {crit }}$ values are 1.042 and $0.461 \AA$. As $q$ further increases, the critical dipole moment seems to approach a limiting value of ca. $2 \mathrm{D}$ (see Fig. 1), which provides an estimate for the critical value $\left(\mu_{c r i t-p d}^{2 e}\right)$ of the point dipole required to bind two electrons as obtained within our limited-basis variational approach and using our $1 \mathrm{~cm}^{-1}$ criterium for critical binding. The small differences among the numerically determined "asymptote" of ca. 2 D and the exact critical value of $1.625 \mathrm{D}$ required to infinitesimally bind one electron, $\mu_{\text {crit }}^{1 e}$, as well as our $q=10,000$ finite basis set one-electron $1 \mathrm{~cm}^{-1}$ critical dipole of $1.76 \mathrm{D}$ raise the question of whether the twoelectron case actually has a critical point dipole

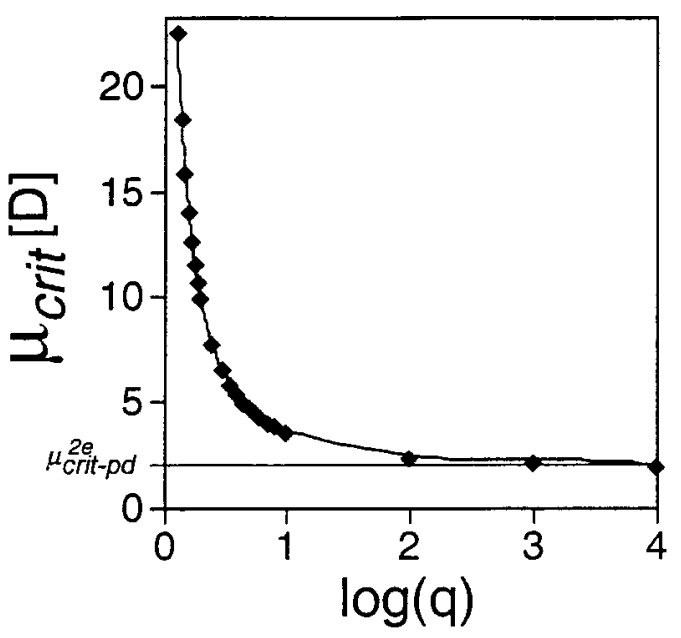

FIGURE 1. Dependence of the critical value of the finite dipole moment $(+q) \stackrel{R}{\cdots}(-q)$, required to bind two electrons, on the charge $q$ of the constituting monopoles. 
moment identical to that of the one-electron case. Because basis set incompleteness effects are expected to be more profound for the two- than for the one-electron system, the difference between $\mu_{c r i t-p d}^{2 e}$ and $\mu_{c r i t}^{1 e}$ for the point dipole case is expected to further decrease with basis set improvements. The question of whether this difference is actually zero or only the inequality $\mu_{c r i t}^{1 e}<\mu_{c r i t-p d}^{2 e}$ $<2 \mathrm{D}$ holds needs to be addressed, which we now do.

Considering the two-electron FFD Hamiltonian

$$
\begin{aligned}
H= & -\frac{1}{2} \nabla_{1}^{2}-\frac{1}{2} \nabla_{2}^{2}-\frac{q}{r_{1}}-\frac{q}{r_{2}}+\frac{q}{\left|\bar{r}_{1}-\bar{R}\right|} \\
& +\frac{q}{\left|\bar{r}_{2}-\bar{R}\right|}+\frac{1}{r_{12}}
\end{aligned}
$$

and scaling the electrons' radial coordinates $r_{i}=$ $\rho_{i} / q$ allows us to rewrite $H$ as

$$
\begin{aligned}
H= & q^{2}\left[-\frac{1}{2} \nabla_{1}^{2}-\frac{1}{2} \nabla_{2}^{2}-\frac{1}{\rho_{1}}-\frac{1}{\rho_{2}}+\frac{1}{\left|\bar{\rho}_{1}-q \bar{R}\right|}\right. \\
& \left.+\frac{1}{\left|\bar{\rho}_{2}-q \bar{R}\right|}+\frac{1}{q} \frac{1}{\rho_{12}}\right]
\end{aligned}
$$

with $\nabla_{i}^{2}$ now referring to $\bar{\rho}_{i}$ rather than to $\bar{r}_{i}$. The solution of the Schrödinger equation $h_{2} \psi_{h}=\epsilon_{h} \psi_{h}$, with $h_{2}=H / q^{2}$, in the limit $q \rightarrow \infty, q \bar{R}=\bar{\mu}$ (finite) have energies $\epsilon_{h}$ that are the sums of the energies of the one-electron Hamiltonian

$$
h_{1}=-\frac{1}{2} \nabla^{2}-\frac{1}{\rho}+\frac{1}{|\bar{\rho}-\bar{\mu}|} .
$$

The eigenvalues of $h_{1}$ are, of course, the energies of the one-electron FFD problem corresponding to a dipole moment $\bar{\mu}$. So, clearly, in the limit $(q \rightarrow \infty$, $R \rightarrow 0, q R$ finite) where the FFD potential approaches the point dipole, the energies of $h_{2}$ approach sums of energies of the one-electron $h_{1}$ and, thus, the critical dipole of $h_{1}$ will also produce critical binding for $h_{2}$. We should, however, stress that even though the conditions for infinitesimal critical binding (1.625 D) for $h_{1}$ and $h_{2}$ are the same as the point dipole limit is reached, it is not true that the conditions for achieving $\approx 1 \mathrm{~cm}^{-1}$ binding in the FFD (or its variant introduced in the next subsection) will be identical for the one- and two-electron cases (also see the last sentence of the subsection Binding of Two Electrons by a Positive $+q$ Charge).

\section{THE FFD-PLUS-CORE MODEL}

Earlier experience on the binding of one electron to FFD potentials showed, when $\mu$ exceeds $\mu_{\text {crit }}$, that the electron binding energies predicted by this model substantially exceed the binding energies of real molecules having the same dipole moment. For example, modeling $\mathrm{LiH}$ using $R$ equal to the equilibrium bond length $\left(R_{e}=1.61 \AA\right)$ and selecting $q=0.77 e$ to have $q R$ properly reproduce the correct dipole moment of $\mathrm{LiH}(\mu=5.95 \mathrm{D})$ produces, using the FFD model and the basis set employed here, a binding energy for one electron of $2.04 \mathrm{eV}$. Realistic ab initio calculations on $\mathrm{LiH}^{-}$ and $\mathrm{LiH}$ using a 6-311G [supplemented with 6( $s p)$ set of diffuse functions] basis set give a binding energy of $0.28 \mathrm{eV}$, much less than that obtained by the FFD model. Clearly, the finite dipole potential overestimates the binding energy because it does not insist that the wave function of the extra electron be excluded from the region occupied by the Li's 1 s core orbitals.

To examine the corresponding effects on the critical binding conditions for two electrons, we carried out calculations in which the $+1 e$ and $-1 e$ charges of the FFD model were replaced by $+3 e /-1 e,+11 e /-1 e$, and $+19 e /-1 e$ with two $\left(1 s^{2}\right), 10\left(1 s^{2} 2 s^{2} 2 p^{6}\right)$, or $18\left(1 s^{2} 2 s^{2} 2 p^{6} 3 s^{2} 3 p^{6}\right)$ electrons located on the positive charge center. ${ }^{\S} \mathrm{A}$ 6-311G/6( $s p)$ basis set was used to describe the core orbitals of these $\mathrm{Li}^{+}, \mathrm{Na}^{+}$, and $\mathrm{K}^{+}$ions, and the SCF procedure was used to optimize these core orbitals. Subsequently, the one- and two-electron full CI method was used to calculate the energies of the singly and doubly charged anion systems. Finally, the position $(R)$ of the $-1 e$ charge was varied to determine the distance $R_{\text {crit }}$ (and, thus, $\mu_{\text {crit }}=1 e R_{\text {crit }}$ ) at which the dianion becomes barely stable. ${ }^{\dagger}$

The critical distances $\left(R_{\text {crit }}=23.53,26.93\right.$, and $29.34 \AA$ ) and neutral dipole moments ( $\mu_{\text {crit }}=113$, 129 , and $141 \mathrm{D})$ thereby obtained for the species where the $+1 e$ charge is replaced by a $\mathrm{Li}^{+}, \mathrm{Na}^{+}$, or $\mathrm{K}^{+}$case are considerably larger than what we find for the corresponding $+1 e /-1 e$ FFD model

\footnotetext{
${ }^{\S}$ A similar model was first used in a study of the relationship between dipole moments and electron binding energies in [28].

${ }^{\dagger}$ Realizing that we are carrying out numerical calculations using a finite basis set and keeping in mind that we are trying to determine under what conditions a molecule can bind two electrons by an amount that is small yet experimentally observable, we chose to define the binding limit to be $1 \mathrm{~cm}^{-1}$ or more when we consider it bound.
} 
( $R_{\text {crit }}=19.19 \AA, \mu_{\text {crit }}=92.17 \mathrm{D}$, see Table I). This is consistent with the $+1 e /-1 e$ model overestimating the binding energy (and thus underestimating $R_{\text {crit }}$ and $\mu_{\text {crit }}$ ) in the two-electron case. These findings suggest that it will be very difficult to find a realistic molecular system capable of binding two electrons through its dipole potential by ca. $1 \mathrm{~cm}^{-1}$.

Finally, we found that the simple approximation to $R_{\text {crit }}=q / E A(q)$, applied to the $\mathrm{Li}^{+}, \mathrm{Na}^{+}$, $\mathrm{K}^{+} /-1 e$ cases described above by using the $E A$ values of $\mathrm{Li}, \mathrm{Na}$, and $\mathrm{K}$ and taking $q=1 e$, gives $R_{\text {crit }}=23.59,27.00$, and $29.41 \AA$ and, thus, $\mu_{\text {crit }}=$ $113.31,129.69$, and $141.26 \mathrm{D}$, respectively, which agree remarkably well with the ab initio values reported above.

\section{Discussion}

We have examined a model system consisting of two electrons moving in the electrostatic potential of two point charges $+q e$ and $-q e$ separated by the distance $R$. We found that the critical value of the finite dipole $\mu_{\text {crit }}=q R_{\text {crit }}$ required to bind two electrons by $\approx 1 \mathrm{~cm}^{-1}$ strongly depends on $q$, unlike the case when one electron is bound to the same potential. In fact, the critical dipole moment for two-electron binding tends to infinity for $q \approx$ $0.91 e$. As $q$ increases and approaches infinity, the finite dipole approaches the point dipole, and our computed critical value $\mu_{\text {crit }}$ for $\approx 1 \mathrm{~cm}^{-1}$ binding approaches a value less than $2 \mathrm{D}$. The value that we achieve for the critical dipole needed to bind one electron $(1.75 \mathrm{D})$ by $>1 \mathrm{~cm}^{-1}$ is close to this two-electron critical value and to the analytical (1.625 D) and earlier (2.0 D) numerical one-electron critical dipoles for infinitesimal binding. Based on our analysis of the q-dependence of the twoelectron FFD Hamiltonian, we showed that the difference in critical dipoles required to bind one and two electrons approaches zero as the point dipole potential is reached.

Overall, our findings suggest that it will be difficult to find a neutral polar molecule which can bind two electrons via its dipole potential. Even for the species in which formally doubly or triply charged atomic ions appear (e.g., in $\mathrm{MgO}$ or $\mathrm{AlN}$ ), the actual bond polarity is seldom consistent with $q \geq 1.5 e$. The FFD model suggests that (see Table I) $\mu_{\text {crit }} \approx 16 \mathrm{D}$ in this case. Moreover, the effects of inner-shell orbitals, as shown in the subsection The
FFD-Plus-Core Model, would cause $\mu_{\text {crit }}$ to be even larger than this $16 \mathrm{D}$ to achieve two-electron binding. Although binding two electrons via the dipole potential of a real molecule may be improbable, it may be possible to form a mixed valencedipole-bound dianion of a polar molecule, with one excess electron dipole-bound and localized on the positive side of molecular dipole and the second excess electron bound by valence interactions to a functional group such as $-\mathrm{CC},-\mathrm{NO}_{2}$, - $\mathrm{COO}$, etc., at a distant part of the molecule. Work in this direction is in progress in our group.

\section{ACKNOWLEDGMENTS}

This work was supported by the NSF Grant CHE9618904 to J. S. and the Polish State Committee for Scientific Research (KBN) Grant No. 3 T09A 04915 (Contract No. 0337/T09/98/15) to P. S. and M. G. The computer time provided by the Utah High Performance Computing Center is also gratefully acknowledged. We also acknowledge support of this work by the Divisions of Geosciences and Chemical Sciences both of the Office of Basic Energy Sciences, U.S. Department of Energy. Pacific Northwest National Laboratory is operated for the U.S. Department of Energy by Battelle Memorial Institute under Contract No. DE-AC6-76RLO 1830.

\section{References}

1. (a) Fermi, E.; Teller, E. Phys Rev 1947, 72, 399. (b) Wightman, A. S. Phys Rev 1949, 77, 521. (c) Wallis, R. F.; Herman, R.; Milnes, H. W. J Mol Spectrosc 1960, 4, 51. (d) Turner, J. E.; Anderson, V. E.; Fox, K. Phys Rev 1968, 174, 81.

2. Crawford, H. O. Proc R Soc (Lond) 1967, 91, 279.

3. Crawford, H. O.; Dalgarno, A. Chem Phys Lett 1967, 1, 23.

4. Brown, W. B.; Roberts, R. E. J Chem Phys 1967, 46, 2006.

5. Jordan, K. D.; Luken, W. J Chem Phys 1976, 64, 2760.

6. Koopmans, T. Physica (Amsterdam) 1934, 1, 104.

7. Gutsev, G. L.; Nooijen, M.; Bartlett, R. J. Phys Rev A 1998, 57, 1646.

8. Smith, D. M. A.; Smets, J.; Elkadi, Y.; Adamowicz, L. J Chem Phys 1998, 109, 1238.

9. Gutowski, M.; Skurski, P.; Simons, J.; Jordan, K. D. Int J Quantum Chem 1997, 64, 183.

10. Gutowski, M.; Skurski, P. J Chem Phys 1997, 107, 2968.

11. Gutowski, M.; Skurski, P. J Phys Chem B 1997, 101, 9143.

12. Gutowski, M.; Jordan, K. D.; Skurski, P. J Phys Chem A 1998, 102, 2624.

13. Skurski, P.; Gutowski, M. J Chem Phys 1998, 108, 6303.

14. Compton, R. N. In Negative Ions (Multiply Charged Negative Ions); Esaulov, V. A., Ed.; Cambridge University: London, 1995. 


\section{SKURSKI, GUTOWSKI, AND SIMONS}

15. Scheller, M. K.; Compton, R. N.; Cederbaum, L. S. Science 1995, 270, 1160.

16. Kalcher, J.; Sax, A. F. Chem Rev 1994, 94, 2291.

17. Freeman, G. F.; March, N. H. J Phys Chem 1996, 102, 4331.

18. Boldyrev, A. I.; Gutowski, M.; Simons, J. Acc Chem Res 1996, 29, 497.

19. Boldyrev, A. I.; Simons, J. J Chem Phys 1992, 97, 2826.

20. Gutowski, M.; Boldyrev, A. I.; Simons, J.; Rak, J.; Błażejowski, J. J Am Chem Soc 1996, 118, 1173.

21. Kendall, R. A.; Dunning, T. H., Jr.; Harrison, R. J. J Chem Phys 1992, 96, 6796.

22. Gutowski, M.; Simons, J. J Chem Phys 1990, 93, 3874.

23. Huzinaga, S. J Chem Phys 1965, 42, 1293.

24. (a) Pekeris, C. L. Phys Rev 1962, 126, 1470. (b) Lykke, K. R.; Murray, K. K.; Lineberger, W. C. Phys Rev A 1991, 43, 6104.
25. Moore, C. E. Table of Atomic Energy Levels, National Bureau of Standards; U.S. GPO: Washington, DC, 1971.

26. McLean, A. D.; Chandler, G. S. J Chem Phys 1980, 72, 5639; Krishnan, R.; Binkley, J. S.; Seeger, R.; Pople, J. A. J Chem Phys 1980, 72, 650.

27. Frisch, M. J.; Trucks, G. W.; Schlegel, H. B.; Gill, P. M. W.; Johnson, B. G.; Robb, M. A.; Cheeseman, J. R.; Keith, T.; Petersson, G. A.; Montgomery, J. A.; Raghavachari, K.; Al-Laham, M. A.; Zakrzewski, V. G.; Ortiz, J. V.; Foresman, J. B.; Cioslowski, J.; Stefanov, B. B.; Nanayakkara, A.; Challacombe, M.; Peng, C. Y.; Ayala, P. Y.; Chen, W.; Wong, M. W.; Andres, J. L.; Replogle, E. S.; Gomperts, R.; Martin, R. L.; Fox, D. J.; Binkley, J. S.; Defrees, D. J.; Baker, J.; Stewart, J. P.; Head-Gordon, M.; Gonzalez, C.; Pople, J. A. Gaussian 94, Revision B.1; Gaussian: Pittsburgh, PA, 1995.

28. Jordan, K. D. J Chem Phys. 1977, 66, 3305. 